\title{
Effect of deformation on two-neutrino double beta decay matrix elements
}

\author{
R. Álvarez-Rodríguez, ${ }^{1}$ P. Sarriguren, ${ }^{1}$ E. Moya de Guerra,${ }^{1}$ \\ L. Pacearescu, ${ }^{2}$ A. Faessler, ${ }^{2}$ F. Simkovic ${ }^{2,3}$ \\ ${ }^{1}$ Instituto de Estructura de la Materia, Consejo Superior de \\ Investigaciones Científicas, Serrano 123, E-28006 Madrid, Spain \\ ${ }^{2}$ Institut für Theoretische Physik, Universität Tübingen, \\ D-72076 Tübingen, Germany \\ ${ }^{3}$ Department of Nuclear Physics, Comenius University, \\ SK-842 15 Bratislava, Slovakia
}

August 19, 2018

\begin{abstract}
We study the effect of deformation on the two-neutrino double beta decay for ground state to ground state transitions in all the nuclei whose half-lives have been measured. Our theoretical framework is a deformed QRPA based in Woods-Saxon or Hartree-Fock mean fields. We are able to reproduce at the same time the main characteristics of the two single beta branches, as well as the double beta matrix elements. We find a suppression of the double beta matrix element with respect to the spherical case when the parent and daughter nuclei have different deformations.
\end{abstract}

It has been observed that the matrix elements $M_{2 \nu}$ in all the measured $2 \nu \beta \beta$ cases for the $0^{+} \rightarrow 0^{+}$ transition are quenched with respect to those predicted from pure quasiparticle transitions. The physical mechanisms responsible for this reduction have been a subject of great interest. In this work, we study the effect of the parent and daughter deformations on the $M_{2 \nu}$ matrix elements.

We use a deformaed quasiparticle random phase approximation (pnQRPA) to describe simultaneously the energy distributions of the single $\beta$ Gamow-Teller (GT) strength and the matrix element $M_{2 \nu}$ of all the double beta emitters whose half-lives have been measured: ${ }^{48} \mathrm{Ca},{ }^{76} \mathrm{Ge},{ }^{82} \mathrm{Se},{ }^{96} \mathrm{Zr},{ }^{100} \mathrm{Mo}$, ${ }^{116} \mathrm{Cd},{ }^{128} \mathrm{Te},{ }^{130} \mathrm{Te},{ }^{136} \mathrm{Xe}$, and ${ }^{150} \mathrm{Nd}$. The formalism includes a deformed quasiparticle basis and residual spin-isospin separable interactions in both particle-hole (ph) and particle-particle (pp) channels. We consider two different deformed mean fields: a phenomenological Woods-Saxon (WS) potential and a selfconsistent Hartree-Fock (HF) with Skyrme interactions [1. While the deformation in the HF calculation is obtained selfconsistently, in the WS case it is an input parameter taken to reproduce the experimental quadrupole moments.

We describe the $2 \nu \beta \beta$ process as two successive GT transitions via intermediate $1^{+}$states,

$$
M_{\mathrm{GT}}^{2 \nu}=\sum_{K=0, \pm 1} \sum_{m_{i}, m_{f}} \frac{\left\langle 0_{f}^{+}\left\|\sigma_{K} t^{-}\right\| \omega_{K}^{m_{f}}\right\rangle\left\langle\omega_{K}^{m_{f}} \mid \omega_{K}^{m_{i}}\right\rangle\left\langle\omega_{K}^{m_{i}}\left\|\sigma_{K} t^{-}\right\| 0_{i}^{+}\right\rangle}{\left(\omega_{K}^{m_{f}}+\omega_{K}^{m_{i}}\right) / 2} .
$$



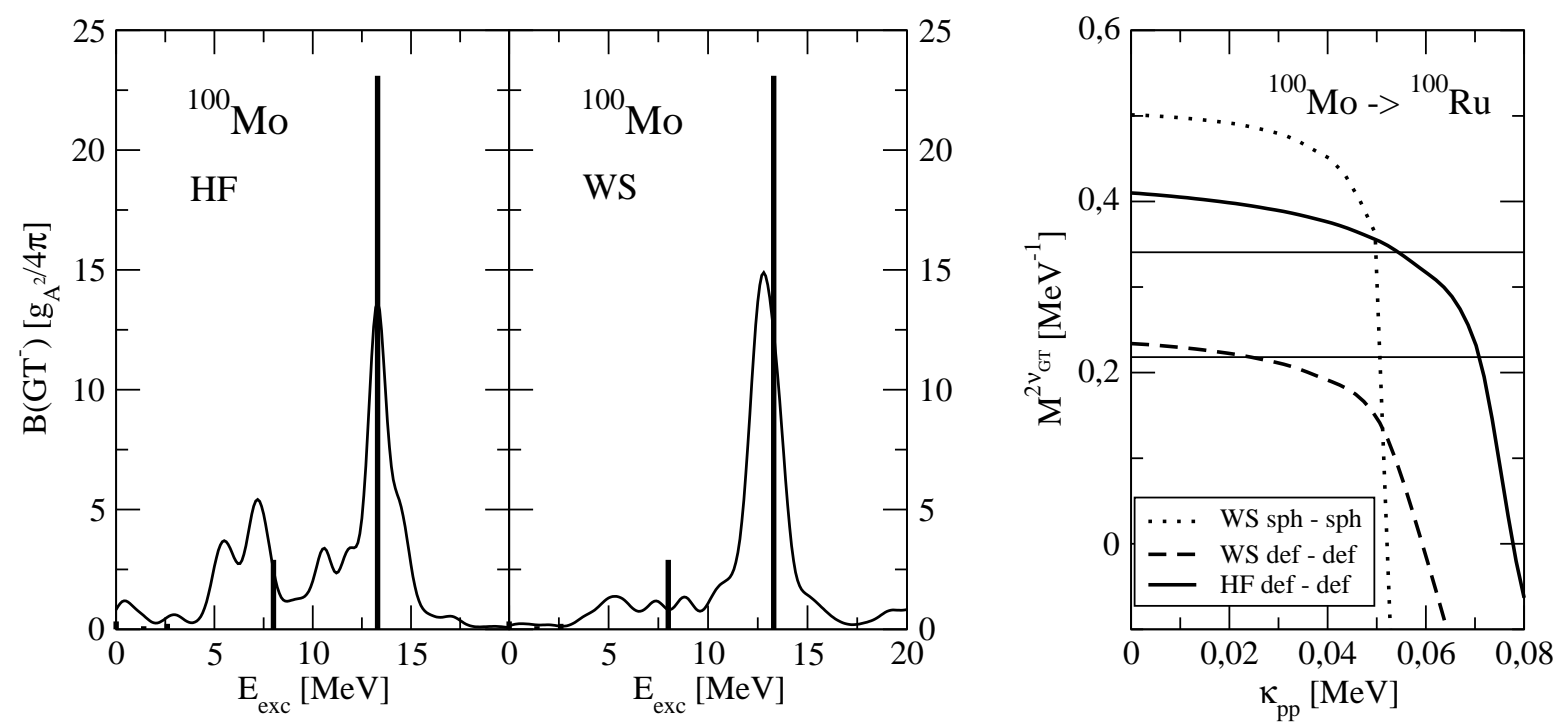

Figure 1: Left panel: $\mathrm{GT}^{-}$strength distributions in ${ }^{100} \mathrm{Mo}$ using HF and WS mean fields. Data (vertical lines) are from Ref. [4]. Right panel: $2 \nu \beta \beta$-decay matrix element as a function of the pp interaction strength. Horizontal lines are the experimental $M_{G T}^{2 \nu}$ values extracted from Ref. [5] using $g_{A}=1.0$ and $g_{A}=1.25$.

The overlap is necessary to take into account the non-orthogonality of the intermediate $1^{+}$states reached from both parent and daughter nuclei [2].

We study first the single GT strength distributions and compare them to the experimental data available. Then, we study the sensitivity of the matrix elements $M_{2 \nu}^{G T}$ to the nuclear deformation and to the pp strength, which is known to be a suppression mechanism. In the case of HF we reproduce simultaneously this information using coupling strengths $\chi_{G T}^{p h}=0.1 \mathrm{MeV}$ and $\kappa_{G T}^{p p}=6 / A \mathrm{MeV}$, while in the case of WS we find that the parametrization of Homma [3], $\chi_{G T}^{p h}=5.2 / A^{0.7} \mathrm{MeV}$ and $\kappa_{G T}^{p p}=0.58 / A^{0.7}$ $\mathrm{MeV}$, reproduces better the experiment. As an example, we show in Fig. 1 the results for ${ }^{100} \mathrm{Mo}$. We notice that deformation introduces a reduction factor with respect to the spherical result. This reduction is larger as the difference between the deformation of parent and daughter increases.

This result is an important starting point to test the validity of this kind of nuclear structure calculations and to tackle the $0 \nu \beta \beta$ decay.

This work was supported in part by MEC (Spain) under contract number BFM2002-03562. One of us (R.A.R.) thanks Ministerio de Educación y Ciencia (Spain) for financial support.

\section{References}

[1] R. Álvarez-Rodríguez, P. Sarriguren, E. Moya de Guerra, L. Pacearescu, A. Faessler and F. Šimkovic, Phys. Rev. C 70 (2004) 064309

[2] F. Šimkovic, L. Pacearescu and A. Faessler, Nucl. Phys. A 733 (2004) 321

[3] H. Homma, E. Bender, M. Hirsch, K. Muto, H.V. Klapdor-Kleingrothaus and T. Oda, Phys. Rev. C 54 (1996) 2972

[4] H. Akimune et al, Phys. Lett. B 394 (1997) 23

[5] A. S. Barabash, Czech. J. Phys. 52 (2002) 567 